\title{
Low-Threshold Lasing and Purcell Effect in Microdisk Lasers at Room Temperature
}

\author{
Toshihiko Baba, Member, IEEE, and Daisuke Sano
}

\begin{abstract}
In this paper, we discuss the Purcell effect, which enhances the spontaneous emission rate, in microdisk lasers operating at room temperature by continuous wave photopumping. We theoretically analyzed the Purcell effect at room temperature by using the four-level rate equations that include the intraband relaxation and the nonradiative effect. We also fabricated $1.55-\mu \mathrm{m}$ GaInAsP microdisk lasers with a minimum diameter of $1.7 \mu \mathrm{m}$ and a minimum threshold power of $19 \mu \mathrm{W}$. Then, we measured the carrier lifetime in a 2.6- $\mu \mathrm{m}$-diameter device by the phase-resolved spectroscopy method, and confirmed that the carrier lifetime was shortened to 1/10 of that in the as-grown epitaxial wafer at a low pump level. From the comparison between the theory and the experiment, we estimated the Purcell factor to be 6.7, the spontaneous emission factor to be 0.11 , and the nonradiative lifetime to be $4 \mathrm{~ns}$. The nonradiative lifetime was consistent with that estimated by another methods. We believe that this is the first demonstration of the Purcell effect in semiconductor microcavities at room temperature.
\end{abstract}

Index Terms-GaInAsP/InP, lifetime measurement, microdisk, phase-resolved spectroscopy, Purcell effect, semiconductor laser, spontaneous emission control, surface recombination.

\section{INTRODUCTION}

$\mathbf{M}$ ICROCAVITY lasers are expected to realize an extremely high efficiency and high speed by the volume effect and the spontaneous emission control. In 1946, Purcell predicted that mode density will be increased and the spontaneous emission rate will be enhanced (i.e., the spontaneous emission lifetime will be shortened) in a microcavity with a high $Q$ and a small volume $V$ [1]. The rate of this lifetime shortening $F$, which is proportional to $Q / V$, is called Purcell factor, and has been discussed by many authors [2]-[4]. Recently, $F=100-155$ was experimentally evaluated for vertical cavity surface emitting lasers, microdisks, and photonic crystals fabricated into GaAs compound materials [5], [6]. However, these experiments were carried out at a cryogenic temperature, and the relation between the Purcell effect and light-emission characteristics was not clearly discussed. Since the nonradiative process is negligible and spectral broadening is sufficiently suppressed at a cryogenic temperature, the interaction between

\footnotetext{
Manuscript received February 18, 2003; revised July 7, 2003. This work was supported in part by the Grant-in Aid 13305009, Nano-Photonic and Electron Devices Technology Project, Focused Research and Development Project for the Realization of the World's Most Advanced IT Nation, and the 21st COE for Creation of Future Social Infrastructure Based on Information Telecommunication Technology, all from the Ministry of Education, Culture, Sports, Science and Technology and in part by CREST 530-13 of the Japan Science and Technology Corporation.

The authors are with the Department of Electrical and Computer Engineering, Yokohama National University, Hodogayaku, Yokohama 240-8501, Japan (e-mail: baba@ynu.ac.jp).

Digital Object Identifier 10.1109/JSTQE.2003.819464
}

a single electron and a photon with an enhanced mode density can be ideally investigated. On the other hand, the nonradiative process increases, which significantly changes the emission characteristic at room temperature. A meaningful evaluation is difficult in GaAs-based microlight emitters, since the surface recombination is dominant at room temperature. Furthermore, a complicated process including a wide spectral broadening and the intraband relaxation of electrons should come into the picture.

In this study, we investigated these phenomena using GaInAsP microdisk lasers emitting at a wavelength $\lambda$ of $1.55 \mu \mathrm{m}$ [7]-[10]. The microdisk strongly confines the whispering gallery mode (WGM) in the disk active layer by the total internal reflection at semiconductor/air boundaries. The size reduction of this cavity is easy, due to its simple geometry and high $Q$ factor. Moreover, the GaInAsP compound materials allow a low-threshold lasing and the Purcell effect at room temperature, since the surface recombination velocity $v_{\mathrm{s}}$ of GaInAsP is one to two orders of magnitude lower than that of GaAs [4], [11]-[13]. So far, we have reported low-threshold continuous wave $(\mathrm{CW})$ lasing in a current-injection device [14] and in a photopumped device [15]. Also, we have evaluated the spontaneous emission factor $C$ by the simple curve-fitting method, where the Purcell effect was neglected [16]. In this study, we theoretically analyzed the GaInAsP microdisk laser using the four-level rate equations including the intraband relaxation [17], [18] and the nonradiative effect. In the experiment, we reduced the device size to a smallest limit determined by the diffraction loss of the WGM, and reduced the threshold. Furthermore, using the phase-resolved spectroscopy, [11], [19] we measured the carrier lifetime of an epitaxial wafer, a mesa, and a microdisk. From the comparison between the theory and the experiment, we evaluated the Purcell factor, the spontaneous emission factor, the nonradiative lifetime, and the lasing threshold at room temperature, and checked their consistency. This paper describes the rate-equation analysis in Section II, the experiment in Section III, and discusses the correspondence between them in Section IV.

\section{RATE-EQUATION ANALYSIS}

\section{A. Formalisms and Solutions}

To take into account the spectral broadening at room temperature, we consider the carrier density $N_{1}$ (the number of careers divided by the active region volume) in the homogeneous broadening spectrum, which contributes to the laser mode, and 
another carrier density $N_{2}$ outside of the homogeneous broadening spectrum. It is considered that $N_{1}$ relax with a short spontaneous emission lifetime $\tau_{\mathrm{sp}}^{\prime}$ by the Purcell effect and $N_{2}$ with the normal lifetime $\tau_{\mathrm{sp}}$. Then, the four-level rate equations on $N_{1}, N_{2}$, and the photon density $S$ (the number of photons divided by the active region volume) of the laser mode are expressed as [17], [18]

$$
\begin{aligned}
\frac{d N_{1}}{d t}= & U P-\frac{c}{n_{\mathrm{g}}} \Gamma_{\mathrm{a}} G S\left(N_{1}-N_{0}\right)-\left(\frac{1}{\tau_{\mathrm{sp}}{ }^{\prime}}+\frac{1}{\tau_{\mathrm{nr}}}\right) N_{1} \\
& -\frac{(1-U) N_{1}-U N_{2}}{\tau_{\mathrm{in}}} \\
\frac{d N_{2}}{d t}= & (1-U) P-\left(\frac{1}{\tau_{\mathrm{sp}}}+\frac{1}{\tau_{\mathrm{nr}}}\right) N_{2} \\
& +\frac{(1-U) N_{1}-U N_{2}}{\tau_{\mathrm{in}}} \\
\frac{d S}{d t}= & \frac{c}{n_{\mathrm{g}}} \Gamma_{\mathrm{a}} G S\left(N_{1}-N_{0}\right)+\frac{C^{\prime}}{U} \frac{N_{1}}{\tau_{\mathrm{sp}}}-\frac{S}{\tau_{\mathrm{ph}}}
\end{aligned}
$$

where $P$ is the pump rate density, $c$ the light velocity in vacuum, $n_{\mathrm{g}}$ is the group refractive index, $\Gamma_{\mathrm{a}}$ is the confinement factor of the laser mode energy into the active region, $G$ is the linear gain coefficient, $N_{0}$ is the transparent carrier density, $\tau_{\mathrm{nr}}$ is the nonradiative recombination lifetime, and $\tau_{\mathrm{ph}}$ is the photon lifetime. $C^{\prime}$ is the spontaneous emission factor defined by the ratio of the spontaneous emission energy coupled into the laser mode to the total spontaneous emission energy. This definition is the same as that for $C$, [20], [21] but we use here a new symbol $C^{\prime}$ to emphasize that it is determined with the influence of the Purcell effect.

The last term in (1) and (2) expresses the intraband relaxation under the thermal equilibrium condition. $\tau_{\text {in }}$ is the intraband relaxation lifetime. It is typically 0.3 ps for GaInAsP [22], [23]. $U$ is the ratio of the homogeneous broadening to the whole spectral broadening $\Delta E$. Using the rectangular spectrum approximation, it is simply expressed as [17], [18]

$$
U=\frac{\frac{2 \hbar}{\tau_{\text {in }}}}{\Delta E}
$$

For $1.55-\mu \mathrm{m}$ GaInAsP compressively strained quantum-wells (CS-QWs), which were used in the experiment of this study, the photoluminescence spectrum exhibited an energy broadening of $36 \mathrm{meV}$ (or a wavelength broadening $\Delta \lambda$ of $70 \mathrm{~nm}$ ). The simplest approximation is to use this broadening as $\Delta E$. We consider that this approximation can still be used in a microdisk, when the Purcell effect is not so strong as to change equilibrium condition between $N_{1}$ and $N_{2}$ and change the spectral broadening. Then, $U$ is calculated to be 0.12 for $\tau_{\text {in }}=0.3 \mathrm{ps}$.

Steady-state solutions $(d / d t=0)$ of (1)-(3) are analytically obtained as (5)-(8) as follows:

$$
S \cong \frac{-\left(\frac{1}{\tau_{4}}-\frac{\tau_{2} c \Gamma_{\mathrm{a}} G P}{n_{\mathrm{g}}}\right)+\sqrt{\left(\frac{1}{\tau_{4}}-\frac{\tau_{2} c \Gamma_{\mathrm{a}} G P}{n_{\mathrm{g}}}\right)^{2}+4 \frac{C^{\prime} \tau_{2}^{2} c \Gamma_{\mathrm{a}} G P}{U \tau_{\mathrm{sp}}^{\prime} \tau_{\mathrm{ph}} n_{\mathrm{g}}}}}{2 \frac{\tau_{2} c \Gamma_{\mathrm{a}} G}{\tau_{\mathrm{ph}} n_{\mathrm{g}}}}
$$

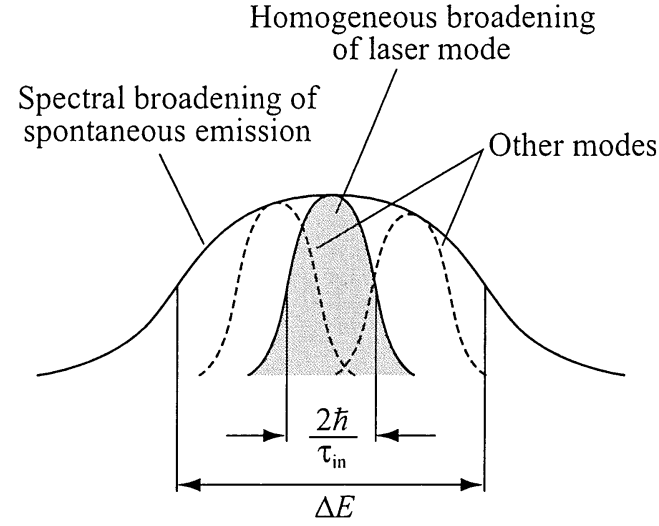

Fig. 1. Relation of the homogeneous broadening of laser mode, those of other modes, and the whole spectral broadening of spontaneous emission.

$$
\begin{aligned}
& N_{1}=\frac{\left\{\left(\tau_{2}-\tau_{3}\right) P+\tau_{2} \frac{c}{n_{\mathrm{g}}} \Gamma_{\mathrm{a}} G N_{0} S\right\}}{\left\{\frac{\tau_{2}}{\tau_{1}}+\frac{\tau_{3}}{\tau_{\mathrm{in}}}+\tau_{2} \frac{c}{n_{\mathrm{g}}} \Gamma_{\mathrm{a}} G S\right\}} \\
& N_{2}=\tau_{3}\left(P+\frac{N_{1}}{\tau_{\text {in }}}\right) \cong \frac{1-U}{U} N_{1}
\end{aligned}
$$

where

$$
\begin{aligned}
& \frac{1}{\tau_{1}}=\frac{1}{\tau_{\mathrm{sp}}}+\frac{1}{\tau_{\mathrm{nr}}} \\
& \frac{1}{\tau_{2}}=\frac{1}{\tau_{\mathrm{sp}}}+\frac{1}{\tau_{\mathrm{nr}}} \\
& \frac{1}{\tau_{3}}=\frac{\frac{1}{\tau_{2}}+\frac{U}{\tau_{\mathrm{in}}}}{1-U} \cong \frac{U}{1-U} \frac{1}{\tau_{\mathrm{in}}} \\
& \frac{1}{\tau_{4}}=\left(\frac{\tau_{2}}{\tau_{1}}+\frac{\tau_{3}}{\tau_{\mathrm{in}}}-\frac{C^{\prime} \tau_{2}}{U \tau_{\mathrm{sp}}}\right) \frac{c}{n_{\mathrm{g}}} \Gamma_{\mathrm{a}} G N_{0}+\frac{1}{\tau_{\mathrm{ph}}}\left(\frac{\tau_{2}}{\tau_{1}}+\frac{\tau_{3}}{\tau_{\mathrm{in}}}\right) .
\end{aligned}
$$

The approximate expression was given by the relation $\tau_{\text {in }} \ll$ $\tau_{\mathrm{sp}}^{\prime}, \tau_{\mathrm{sp}}, \tau_{\mathrm{nr}}$.

\section{B. Lifetime and Spontaneous Emission Factor}

The Purcell effect is expected to enhance the spontaneous emission rate of the laser mode. Therefore, if one directly measures the time decay of the laser mode far below laser threshold, one will directly evaluate the Purcell factor F. On the other hand, if one uses the fitting of a theoretical result of the rate equation analysis to an experimental result, one should evaluate the spontaneous emission lifetime. The spontaneous emission lifetime is defined as the inverse of the spectral integration of the spontaneous emission rate. As shown in Section III, we performed the carrier lifetime measurement and evaluated the spontaneous emission lifetime. Here, the laser mode decay below threshold was not selectively observed but the lifetime was measured over whole the spontaneous emission spectrum. With this background, we discuss here the expression of the spontaneous emission lifetime and the spontaneous emission factor $\mathrm{C}^{\prime}$ with the Purcell factor F.

As shown in Fig. 1, homogeneous broadening spectra of other modes are overlapping with that of the laser mode. Let us use symbol $C$ as the spontaneous emission factor, which is estimated without considering the Purcell effect. Then, the total 
number of allowed cavity modes in the spectral broadening is expressed as $1 / C$ from the simplest interpretation of the spontaneous emission factor [21]. And the number of the modes that overlap with the homogeneous broadening spectrum is expressed as $U / C$. Let us assume that the Purcell factor does not act on other modes but only on the laser mode. That is, only the laser mode has the fast spontaneous emission lifetime $\tau_{\mathrm{sp}} / F$ and other $U / C-1$ modes have the normal spontaneous emission lifetime $\tau_{\mathrm{sp}}$. Suppose that the transition of $N_{1}$ is determined by these lifetimes. The following relations are obtained

$$
\begin{aligned}
\frac{1}{\tau_{\mathrm{sp}}^{\prime}} & =\frac{\frac{U}{C}-1+F}{\frac{U}{C}} \frac{1}{\tau_{\mathrm{sp}}} \cong \frac{U+F C}{U} \frac{1}{\tau_{\mathrm{sp}}} \\
C^{\prime} & =\frac{U F}{\frac{U}{C}-1+F} \cong \frac{U F C}{U+F C} .
\end{aligned}
$$

The approximate expressions were given by $F \gg 1$. Equations (9) and (10) indicate that $\tau_{\mathrm{sp}}^{\prime}$ and $C^{\prime}$ can be approximated as a function of $F C$. For a specified polarization mode, $F$ and $C$ are expressed as [4], [10]

$$
\begin{aligned}
& F=\frac{p \Gamma_{\mathrm{r}} \lambda^{3}}{4 \pi^{2} n^{3} V} Q \\
& C=\frac{p \Gamma_{\mathrm{r}} \lambda^{3}}{4 \pi^{2} n^{3} V} \cdot \frac{\lambda}{\Delta \lambda}
\end{aligned}
$$

where $p$ is the factor denoting the polarization anisotropy in the spontaneous emission, $\Gamma_{\mathrm{r}}$ the relative confinement factor, which expresses the relative intensity of the mode standing wave at the active region, and $n$ the equivalent refractive index of the laser mode. $V$ is the cavity volume, but replaced by the equivalent cavity volume (mode volume) when the mode has a complex spatial distribution. From (11) and (12)

$$
F C=\left(\frac{p \Gamma_{\mathrm{r}} \lambda^{3}}{4 \pi^{2} n^{3} V}\right)^{2} Q \frac{\lambda}{\Delta \lambda} .
$$

\section{Calculation Examples}

Fig. 2 shows the photon density $S$ calculated with the pump rate density normalized by the threshold. Referring to [10], calculation parameters for a GaInAsP CS-QW active layer were determined to be $n_{\mathrm{g}}=3.5, \Gamma_{\mathrm{a}}=0.078, G^{\prime}=6 \times 10^{-16} \mathrm{~cm}^{2}$, $N_{0}^{\prime}=1.5 \times 10^{18} \mathrm{~cm}^{-3}, \tau_{\text {in }}=0.3 \mathrm{ps}, \tau_{\mathrm{sp}}=20 \mathrm{~ns}$, and $\tau_{\mathrm{ph}}=3.5 \mathrm{ps}$. This large $n_{\mathrm{g}}$, which was not used in [10], was assumed here, not only due to the large material dispersion near the band-edge wavelength, but also due to the large structural dispersion in the microdisk. But, we later noticed that the result was less dependent on $n_{\mathrm{g}}$. $G^{\prime}$ and $N_{0}^{\prime}$ are those used for a two-level rate-equation analysis. For the four-level rate equations, they are rectified by the relation $G=G^{\prime} / U$ and $N_{0}=U N_{0}^{\prime}$ [18]. As shown in Fig. 2, the sharp kink of $S$ at the threshold becomes unclear as $F C$ increases and finally approaches the thresholdless characteristic. As $\tau_{\text {nr }}$ decreases, $S$ below the threshold particularly decreases. Such characteristics on $S$ are qualitatively the same as those which do not involve the Purcell effect. Fig. 3 shows the career lifetime $\tau_{1}$ of $N_{1}$ in (8). The lifetime is shortened when $F C$ increases. It is very reasonable that the lifetime is further shortened by a shorter nonradiative lifetime $\tau_{\mathrm{nr}}$.

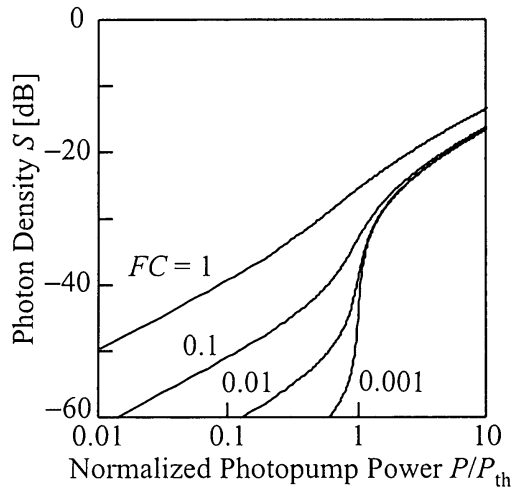

(a)

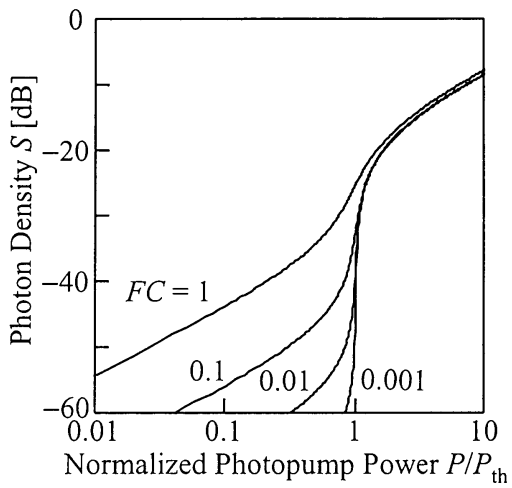

(b)

Fig. 2. Photon density $S$ calculated with the pump power normalized by the threshold. $F C$ is taken as a parameter. (a) $\tau_{\text {nr }}$ is infinity and (b) $4 \mathrm{~ns}$.

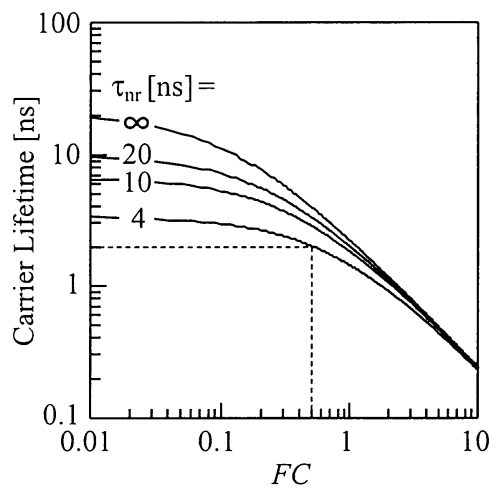

Fig. 3. Carrier lifetime $\tau_{1}$ of $N_{1}$ calculated with $F C$. The nonradiative lifetime $\tau_{\mathrm{nr}}$ is taken as a parameter.

\section{Threshold}

The balance between gain and loss gives the threshold condition

$$
\frac{c}{n_{\mathrm{g}}} \Gamma_{\mathrm{a}} G\left(N_{1 \mathrm{th}}-N_{0}\right)-\frac{1}{\tau_{\mathrm{ph}}}=0
$$

where $N_{1 \text { th }}$ is the threshold carrier density of $N_{1}$. By substituting (6) into (14), the threshold pump-rate density $P_{\mathrm{th}}$ is given by

$$
P_{\mathrm{th}}=\frac{\left(\frac{1-\frac{C \prime}{U}}{\tau_{\mathrm{sp}}}+\frac{1}{\tau_{\mathrm{nr}}}+\frac{\tau_{3}}{\tau_{2} \tau_{\mathrm{in}}}\right)\left(\frac{n_{\mathrm{g}}}{c \Gamma_{\mathrm{a}} G \tau_{\mathrm{ph}}}+N_{0}\right)}{1-\frac{\tau_{3}}{\tau_{2}}} .
$$


One may think that the shortening of the spontaneous emission lifetime increases the threshold with relation $P_{\mathrm{th}}=N_{\mathrm{th}} / \tau_{\mathrm{sp}}$. However, using (9) and (10), and relation $\tau_{\text {in }} \ll \tau_{\mathrm{sp}}^{\prime}, \tau_{\mathrm{sp}}, \tau_{\mathrm{nr}},(15)$ is simplified to

$$
P_{\mathrm{th}} \cong \frac{1}{U}\left(\frac{1-C}{\tau_{\mathrm{sp}}}+\frac{1}{\tau_{\mathrm{nr}}}\right)\left(\frac{n_{\mathrm{g}}}{c \Gamma_{\mathrm{a}} G \tau_{\mathrm{ph}}}+N_{0}\right) .
$$

In (16), effects of $\tau_{\mathrm{sp}}^{\prime}$ and $C^{\prime}$ disappeared, and only the effect of $1-C$ remained. Therefore, the threshold does not increase even by a shorter spontaneous emission lifetime, but rather decreases by a large $C$ factor close to 1 , even without the Purcell effect. This result is equivalent to that derived in [24].

\section{EXPERIMENTS}

\section{A. Device Fabrication and Lasing Characteristics}

In this experiment, we prepared a $1.55-\mu \mathrm{m}$ GaInAsP CS-QW epitaxial wafer. The thickness and the strain of each of the five quaternary QWs were $5 \mathrm{~nm}$ and $1 \%$, respectively. The thickness of $1.20-\mu \mathrm{m}$ quaternary barrier layers was $12 \mathrm{~nm}$. Outside of these layers, there were gradient-index separate confinement heterostructure (GRIN-SCH) layers of 1.20-, 1.15-, and $1.10-\mu \mathrm{m}$ quaternary materials, each of which has $30-\mathrm{nm}$ thickness. These layers were grown on n-type $\left(\sim 2 \times 10^{18} \mathrm{~cm}^{-3}\right)$ InP substrate and covered with p-type $\left(\sim 1 \times 10^{18} \mathrm{~cm}^{-3}\right)$ InP top layer. The active layer was undoped, but it was actually n-type doped of $10^{17}-\mathrm{cm}^{-3}$ order. The fabrication process of the device was the same as that shown in [15]. The circular resist pattern of 1.5-3.0- $\mu \mathrm{m}$ diameters was formed by electron beam lithography, and cylinder mesas were formed by $\mathrm{Cl}_{2} / \mathrm{Xe}$ inductively coupled plasma etching. Finally, the InP above and below the active layer was selectively etched by $\mathrm{HCl}$ solution to form the microdisk supported by a narrow InP pedestal, as shown in Fig. 4.

In the measurement, a semiconductor laser with a wavelength of $0.98 \mu \mathrm{m}$ was used as a photopump source. The light output from a multimode fiber of $100 \mu \mathrm{m}$ in core diameter was focused on a device by an objective lens. The emission from the device was detected by another multimode fiber whose tip was placed $\sim 100 \mu \mathrm{m}$ apart from the device, and analyzed by optical spectrum analyzer. The irradiated light spot was $40 \mu \mathrm{m}$, and its intensity distribution was almost uniform. Therefore, by taking account of the resonant absorption [15], the effective pump power $P_{\mathrm{e}}$ is expressed as $P_{\mathrm{e}}=P_{\mathrm{i}}\left(a^{2} / s^{2}\right)(1-R)(1-$ $\left.e^{-\alpha d}\right) /\left(1-R e^{-\alpha d}\right)$, where $P_{\mathrm{i}}$ is the input power, $a$ is the disk radius, $s$ is the irradiated radius, $R$ is the reflectivity at the disk surface $(\sim 30 \%), \alpha$ is the optical absorption coefficient of the disk $\left(\sim 20000 \mathrm{~cm}^{-1}\right)$, and $d$ is the total thickness of the disk $(=248 \mathrm{~nm})$

Fig. 4 also shows the room temperature $\mathrm{CW}$-lasing characteristic of the device with a diameter of $1.7 \mu \mathrm{m}$. The threshold light power irradiated to the disk (irradiated power) was $60 \mu \mathrm{W}$, and the corresponding absorbed power by the disk (effective pump power) was $19 \mu \mathrm{W}$. This was the lowest value for microdisk lasers so far reported. The lasing spectrum exhibited a single peak with a linewidth determined by the multimode fiber used for detection and the optical spectrum analyzer. Fig. 5 summarizes some data on threshold for current injection and photop-

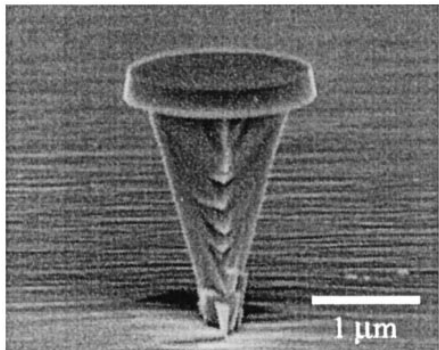

(a)

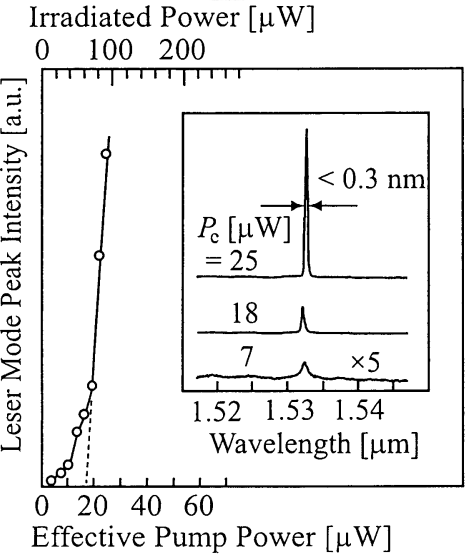

(b)

Fig. 4. (a) Scanning electron microscope image of 1.7- $\mu \mathrm{m}$-diameter device. (b) Laser peak intensity versus light power characteristic at room temperature under $\mathrm{CW}$ condition. The upper axis shows the irradiated power to the disk, and the lower axis shows the effective pump power. Inset shows lasing spectra.

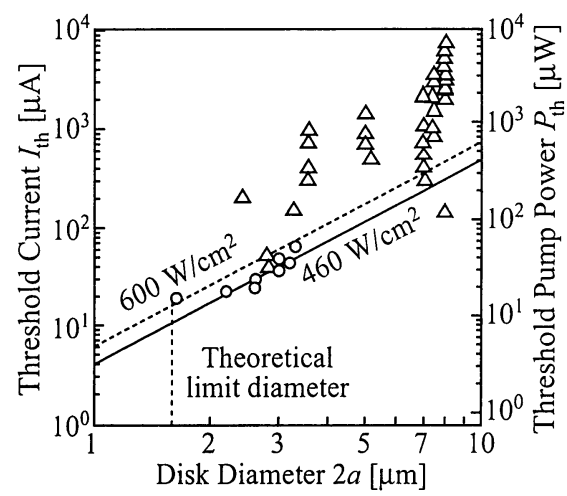

Fig. 5. Threshold power recorded for microdisks with various diameters Triangles denote current injection devices and circles denote photopumped devices.

umped devices with various diameters, which were obtained in this group. In this figure, the threshold current in the injection devices can be directly compared with the threshold power in the photopumped devices, since the right axis is shifted against the left axis by the conversion factor from the current to the power. The threshold values in the injection devices are generally higher than those of photopumped devices, because of the nonuniform carrier distribution [10]. As the disk diameter $2 a$ decreases, the threshold decreases in proportion to $(2 a)^{2}$. When it is $2 a>2 \mu \mathrm{m}$, the threshold values of photopumped devices lie along a threshold density line of $460 \mathrm{~W} / \mathrm{cm}^{2}$. This value is lower than that for long-cavity broad-area lasers of the as-grown 


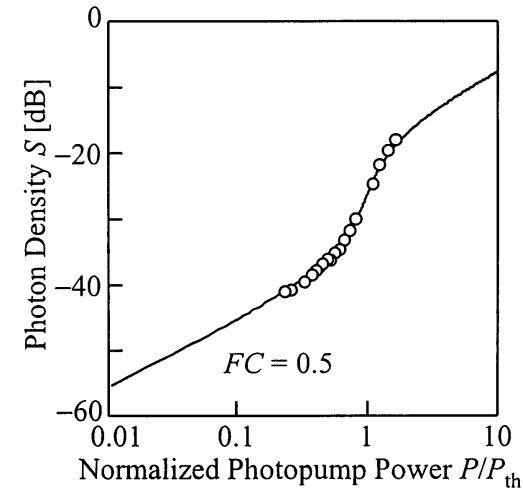

Fig. 6. Laser-mode power with the normalized pump power in a 2.6- $\mu \mathrm{m}$-diameter device at room temperature. It was measured by the setup of the phase resolved spectroscopy. The fitting curve is calculated for a nonradiative lifetime of $4 \mathrm{~ns}$ and $F C=0.5$.

wafer, i.e., $\sim 600 \mathrm{~W} / \mathrm{cm}^{2}$ (it was obtained by converting a measured threshold current density of $\sim 700 \mathrm{~A} / \mathrm{cm}^{2}$ into the power dimension). Such a low threshold density is attributed to the strong optical confinement in the semiconductor/air waveguide of the microdisk. By the way, in Sections III-B and IV-B, we will show that the spontaneous emission lifetime was shortened in a 2.6- $\mu \mathrm{m}$-diameter device. However, a remarkable increase in threshold is not observed for this diameter in Fig. 5. This shows that, as noted in Section II-D, the threshold is not influenced by the shortening of the spontaneous emission lifetime but can, rather, be reduced by factor $1-C$. But it is not clearly observed in Fig. 6 due to a small $C$ factor and a large nonradiative recombination, as discussed in Section IV. For $2 a<2 \mu \mathrm{m}$, the threshold goes up from the threshold density line. It should be affected by the increase in surface recombination at the disk sidewall [11], [12] and/or the increase in scattering loss caused by the imperfection of the disk [26], [27]. The detail of the former issue will be discussed in Section IV. According to a three-dimensional finite difference time domain simulation, the smallest limit diameter of this device for lasing operation is 1.6-1.7 $\mu \mathrm{m}$ in this wavelength range [28]. The minimum device in this study is equal to this limit diameter, so further size reduction may be difficult. In order to avoid the diffraction loss against further size reduction, more complex structures, such as microgears [29], [30] and photonic crystals [31], [32] are needed.

\section{B. Lifetime Measurement}

In the phase-resolved spectroscopy, the device was photopumped by a YAG laser light at $\lambda=1.06 \mu \mathrm{m}$, which was sinusoidally modulated by an electrooptic modulator with frequencies of 1-50 MHz. The time dependency of the emission from a device was measured through a bandpass filter of $1.52-1.58 \mu \mathrm{m}$, which covered the center spectral range of the spontaneous emission. The time dependency of the pump light, which was reflected at the device surface, was also measured. The carrier lifetime was calculated by measuring the difference of the phase and the modulation rate between the two waves. Unlike the time-resolved method, which is widely used for the lifetime measurement, the time-average emission power in this method is three or more orders of magnitude higher, due to the $\mathrm{CW}$ pumping. Therefore, even in the wavelength range of

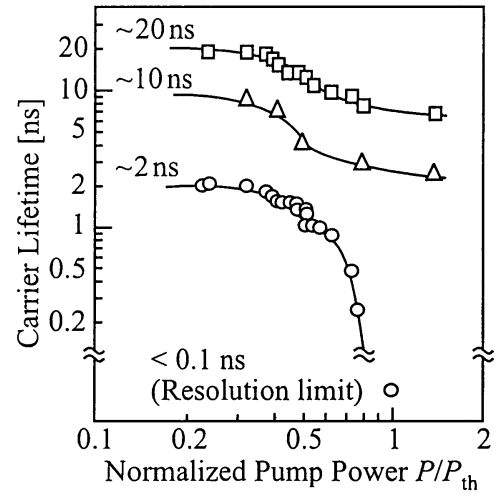

Fig. 7. The carrier lifetime measured with the normalized pump power. Squares denote the as-grown wafer, triangles the mesa, and circles the 2.6- $\mu \mathrm{m}$-diameter microdisk.

1.5-1.6 $\mu \mathrm{m}$, for which we have no photodetectors with a high sensitivity and a fast response time below $1 \mathrm{~ns}$, we can measure the lifetime of weak spontaneous emission from a tiny device such as microdisks. Still, the minimum detected power was limited by the electrical signal noise from the modulator driver. We tried the spectrally resolved measurement for the direct evaluation of the Purcell factor by inserting a monochrometer in the optical path. However, this configuration did not provide sufficient stability of the result below threshold. Therefore, we employed the lifetime evaluation against the whole spontaneous emission spectrum by using the bandpass filter. The shortest lifetime measured in this system depends on the resolution of the phase difference, and it was nearly $0.1 \mathrm{~ns}$ in this experiment.

The smallest diameter device shown in Section III-A was not used for the lifetime measurement because of the degradation. Therefore, we used another device of $2.6 \mu \mathrm{m}$ diameter, which lased at $\lambda=1.550 \mu \mathrm{m}$, as a sample. The lasing characteristic measured in the setup explained in the previous section is shown in Fig. 6. When the same characteristic was measured by the setup for the phase resolved spectroscopy, the spectral peak of the laser mode was only slightly higher than the background spontaneous emission spectrum at a low pump level below threshold. This is because the pumping and detection were carried out from the top direction in the measurement, and the radiation of the laser mode is not efficient in this direction. Such a condition is rather suitable for evaluating the lifetime discussed in Section III-B. Carrier lifetimes measured for the as-grown wafer, a $100 \times 100 \mu \mathrm{m}^{2}$ square mesa, and the microdisk are summarized in Fig. 7. The measurement was also carried out for a smaller mesa with the same diameter as that of the microdisk. But we eliminate the results from Fig. 7, since the emission intensity was too weak to obtain reliable data. The carrier lifetime of the as-grown wafer was $\sim 20 \mathrm{~ns}$ at the lowest pump level and did not change notably with the increase in pump level. Even at the threshold pump level for the microdisk, it was still as long as $10 \mathrm{~ns}$. This small change is considered to be due to the radiative recombination with dopants in the active layer [11]. Although a similar characteristic was observed for the large mesa, the carrier lifetime at the lowest pump level was shortened to $\sim 10 \mathrm{~ns}$. In the microdisk, the carrier lifetime at this level was shortened to $2 \mathrm{~ns}$. Near the threshold pump level, the lifetime decreased rapidly to $<0.1 \mathrm{~ns}$. At such a high pump level, 
the laser mode intensity is much stronger than the background spectrum. Therefore, we cannot evaluate the spontaneous emission lifetime but the stimulated emission in this regime.

\section{EVALUATION}

\section{A. Surface Recombination}

The carrier lifetime $\tau_{\mathrm{C}}$ shown in Section III-B is expressed as

$$
\frac{1}{\tau_{\mathrm{c}}}=\frac{1}{\tau_{\mathrm{sp}}}+\frac{1}{\tau_{\mathrm{nr}}}=\frac{1}{\tau_{\mathrm{sp}}}+\frac{1}{\tau_{\mathrm{ct}}}+\frac{1}{\tau_{\mathrm{sf}}}+\frac{1}{\tau_{\mathrm{ag}}}
$$

where $\tau_{\mathrm{ct}}$ is the nonradiative lifetime by the nonradiative center induced during the epitaxial growth and the fabrication process, $\tau_{\mathrm{sf}}$ is the surface recombination lifetime, and $\tau_{\mathrm{ag}}$ is the Auger recombination lifetime. However, considering a high wafer quality and the low pump level, $\tau_{\mathrm{ct}}^{-1}$ and $\tau_{\mathrm{ag}}^{-1}$ are ignored in the following. The surface recombination lifetime $\tau_{\mathrm{sf}}$ is given by

$$
\frac{1}{\tau_{\mathrm{sf}}}=\frac{v_{\mathrm{sf}}\left(S_{\mathrm{a} 1}+S_{\mathrm{a} 2} e^{-\frac{\Delta E}{k_{\mathrm{B}} T}}\right)}{V_{\mathrm{a}}}
$$

where $v_{\mathrm{sf}}$ is the surface recombination velocity, $S_{\mathrm{a} 1}$ is the exposed sidewall area of the CS-QWs, $S_{\mathrm{a} 2}$ is the surface area of the sample, $\Delta E$ is an energy barrier from the electron energy in the CS-QWs to the sample surface, $k_{\mathrm{B}} T$ is a thermal energy (26 meV at room temperature), and is $V_{\mathrm{a}}$ the volume of the CS-QWs.

The total thickness of the CS-QWs was $23 \mathrm{~nm}$, and the piece of the as-grown wafer used for the lifetime measurement was of square-millimeter-order wide. Since the pump light was irradiated at the center of the wafer, the surface recombination at the sidewall can be neglected. In addition, it had a InP top layer, so $\Delta E$ is estimated to be $210 \mathrm{meV}$ by approximating the electron energy as the quantized energy of the CS-QWs and assuming a band offset of the conduction band to be $40 \%$ of the bandgap energy difference. Since the surface recombination velocity of the same wafer was measured to be $v_{\mathrm{sf}}=1.2 \times 10^{4} \mathrm{~cm} / \mathrm{s}$, [12] $\tau_{\mathrm{sf}}$ at the wafer surface was estimated to be over $500 \mathrm{~ns}$. This value is also negligible, compared with the measured carrier lifetime. Therefore, we can consider that measured lifetime of $20 \mathrm{~ns}$ is equivalent to the spontaneous emission lifetime of the wafer $\tau_{\mathrm{sp}}$. The mesa was still larger than the irradiation area, so the surface recombination at the sidewall is negligible. However, the top InP was removed and the 1.1- $\mu \mathrm{m}$-quaternary GRIN-SCH layer was exposed in the mesa. Then, $\Delta E$ is estimated to be $127 \mathrm{meV}$, the corresponding surface recombination lifetime is $23 \mathrm{~ns}$, and from (17), the carrier lifetime $\tau_{\mathrm{c}}=11 \mathrm{~ns}$. This estimation well agrees with the measured result. For the microdisk, the pump light absolutely covers the microdisk. Therefore, the surface recombination can occur at the sidewall, and $\tau_{\mathrm{sf}}=4.5 \mathrm{~ns}$ and $\tau_{\mathrm{c}}=3.7 \mathrm{~ns}$ are estimated for a disk diameter of $2.6 \mu \mathrm{m}$. This estimation does not agree with the measured value $2 \mathrm{~ns}$. If one explains this discrepancy by the Purcell effect, one has to consider that the spontaneous emission lifetime of the as-grown wafer $20 \mathrm{~ns}$ was shortened to $3.6 \mathrm{~ns}$.

\section{B. Fitting With the Theory}

In the phase-resolved spectroscopy described in Section III-B, we measured the lifetime of carrier density $N_{1}$, i.e., $\tau_{1}$, through the bandpass filter. Therefore, we can uniquely evaluate $F C$ and $\tau_{\text {sf }}$ by fitting theoretical results with measured results without using the discussion in Section IV-A. Figs. 3 and 6 show the results of the fitting. Only when $F C=0.5$ and $\tau_{\mathrm{sf}}=4 \mathrm{~ns}$ were assumed, theoretical results were fitted to all the experiment results in Section III. This $\tau_{\text {sf }}$ is in good agreement with the value evaluated in Section IV-A. It is seen from (9) that $F C=0.5$ corresponds to $\tau_{\mathrm{sp}}^{\prime}=\tau_{\mathrm{sp}} / 5.2$. This is also in good argument with the expectation in Section IV-A. Provided a typical $Q$ factor of 2000 and $\Delta \lambda=70 \mathrm{~nm}$, then $C=0.074$ and $F=6.7$ are estimated from (13), and $C^{\prime}=0.11$ from (10). Assuming the relative containment factor $\Gamma_{\mathrm{r}}[4]$ of 1.5 for the relative position of the CS-QWs and the laser mode, the polarization anisotropy factor $p$ of 1.5 for the TE polarization in the CS-QWs, [10] $\lambda=1.550 \mu \mathrm{m}$, and the equivalent refractive index of 2.65 [4], a mode volume $V=3.4 \mu \mathrm{m}^{3}$ is estimated from (13). This value is nearly four times larger than $0.8 \mu \mathrm{m}^{3}$, which is simply estimated by considering the mode distribution in this device. Inversely speaking, evaluated values of $\mathrm{F}$ and $\mathrm{C}$ are four times smaller than expected values. We consider two reasons. One is the detuning of the laser mode wavelength from the spontaneous emission peak. Although the spontaneous emission peak is $1.53-1.55 \mathrm{~mm}$ in the as-grown wafer, it can be shifted to longer wavelength under the influence of the strain relaxation in the CS-QWs [33]. It has been shown that by the detuning of $50 \mathrm{~nm}, \mathrm{C}$ is reduced four times smaller. The other reason is regarding the measurement of the carrier lifetime. Ideally, the spectral range for the measurement should be limited to the homogeneous broadening of the laser mode so that $\mathrm{t} 1$ is selectively evaluated. But actually, the bandpass filter used in the measurement covered a wider spectral range. In addition, not only the disk edge, at which the laser mode is localized, but also the disk center was photopumped. Therefore, the measured wave included the emission from the disk center. These might weaken the influence of the Purcell effect on the measured lifetime.

\section{CONCLUSION}

The four-level rate equation analysis was carried out and the Purcell effect at room temperature was estimated for GaInAsP microdisk lasers. In the experiment, the room temperature $\mathrm{CW}$ lasing with an effective threshold pump power of $19 \mu \mathrm{W}$ was obtained for the smallest disk diameter $1.7 \mu \mathrm{m}$, which is almost the smallest limit of this type of device. Moreover, the carrier lifetime in a $2.6-\mu \mathrm{m}$-diameter device was measured, and it was found that the lifetime of the device was ten times shorter than of that of the as-grown wafer. From the comparison of theoretical and the experimental results, the Purcell factor of 6.7, the spontaneous emission factor of 0.11 and nonradiative radiative lifetime of 4 ns were estimated. These results showed a good consistency with other estimations. The analysis also indicated that the lasing threshold has no dependency on the Purcell effect. This result is confirmed by checking the experiment data.

So far, the Purcell effect has been evaluated only at cryogenic temperature. Therefore, this study is the first demonstration of this effect in a semiconductor microcavity laser at room temperature. As a future work, the observation of the time decay of laser mode below threshold by a high sensitivity photodetector will allow more direct evaluation of the Purcell factor. A larger 
Purcell factor is expected in a smaller microdisk and/or a single defect photonic crystal laser [31], [32] with a fine control of laser mode. For a very strong Purcell effect, the relation of the intraband relaxation and the carrier dynamics will play a more significant role in the spontaneous emission process. Under such condition, we may not be able to approximate the spectral broadening of the spontaneous emission in the cavity by that of the as-grown wafer. It is an interesting next issue to be investigated. Besides, the nonradiative recombination still exhibited a significant influence in the experimental result. Therefore, further investigation on its reduction method such as that described in [12] is desirable.

\section{ACKNOWLEDGMENT}

The authors would like to thank Prof. Y. Kokubun, Yokohama National University, as well as Prof. K. Iga and Prof. F. Koyama, Tokyo Institute of Technology, for their helpful suggestions.

\section{REFERENCES}

[1] E. M. Purcell, "Spontaneous emission probabilities at radio frequencies," Phys. Rev., vol. 69, p. 681, 1946.

[2] H. Yokoyama and K. Ujihara, Eds., Spontaneous Emission and Laser Oscillation in Microcavities. Boca Raton, FL: CRC, 1995.

[3] C. Weisbuch and J. Rarity, Eds., Microcavities and Photonic Bandgaps: Physics and Applications. Dordrecht, The Netherlands: Kluwer, 1996.

[4] T. Baba, "Photonic crystals and microdisk cavities based on GaInAsP/InP system," IEEE J. Select. Topics Quantum Electron., vol. 3, pp. 808-830, June 1997.

[5] J. M. Gerard and B. Gayral, "Strong Purcell effect for InAs quantum boxes in three-dimensional solid-state microcavities," J. Lightwave Technol., vol. 17, pp. 2089-2095, 1999.

[6] L. A. Graham, D. L. Huffaker, and D. G. Deppe, "Spontaneous lifetime control in a native-oxide-apertured microcavity," Appl. Phys. Lett., vol. 74, pp. 2408-2410, 1999.

[7] S. L. McCall, A. F. J. Levi, R. E. Slusher, S. J. Pearton, and R. A. Logan, "Whispering-gallery mode microdisk lasers," Appl. Phys. Lett., vol. 60, pp. 289-291, 1992.

[8] A. F. J. Levi, S. L. McCall, S. J. Pearton, and R. A. Logan, "Room temperature operation of submicrometer radius disc laser," Electron. Lett., vol. 29, pp. 1666-1667, 1993.

[9] T. Baba, M. Fujita, A. Sakai, M. Kihara, and R. Watanabe, "Lasing characteristics of GaInAsP/InP strained quantum-well microdisk injection lasers with diameter of 2-10 $\mu \mathrm{m}$," IEEE Photon. Technol. Lett., vol. 9, pp. 878-880, July 1997.

[10] M. Fujita, A. Sakai, and T. Baba, "Ultra-small and ultra-low threshold microdisk injection laser-design, fabrication, lasing characteristics and spontaneous emission factor," IEEE J. Select. Topics Quantum Electron., vol. 5, pp. 673-681, 1999.

[11] T. Baba, K. Inoshita, H. Tanaka, J. Yonekura, M. Ariga, A. Matsutani, T. Miyamoto, F. Koyama, and K. Iga, "Strong enhancement of light extraction efficiency in GaInAsP 2-D photonic crystals of columns," $J$. Lightwave Technol., vol. 17, pp. 2113-2120, 1999.

[12] H. Ichikawa, K. Inoshita, and T. Baba, "Reduction in surface recombination of GaInAsP/InP micro-columns by $\mathrm{CH}_{4}$ plasma irradiation," Appl. Phys. Lett., vol. 78, pp. 2119-2121, 2001.

[13] M. Boroditsky, I. Gontijo, M. Jackson, R. Vrijen, E. Yablonovitch, T. Krauss, C.-C. Cheng, A. Scherer, R. Bhat, and M. Krames, "Surface recombination measurements on III-V candidate materials for nanostructure light-emitting diodes," J. Appl. Phys., vol. 87, pp. 3497-3504, 2000.

[14] M. Fujita, R. Ushigome, and T. Baba, "Continuous wave lasing in GaInAsP microdisk injection laser with threshold current of $40 \mu \mathrm{A}$," Electron. Lett., vol. 36, pp. 790-791, 2000.

[15] M. Fujita, K. Teshima, and T. Baba, "Low threshold continuous wave lasing in photo-pumped GaInAsP microdisk lasers," Jpn. J. Appl. Phys., vol. 40, pp. L875-L877, 2001.

[16] M. Fujita, R. Ushigome, and T. Baba, "Large spontaneous emission factor of 0.1 in a microdisk injection laser," IEEE Photon. Technol. Lett., vol. 13, pp. 403-405, May 2001.

[17] K. Furuya, Y. Suematsu, and Y. Sakakibara, "Influence of intraband electronic relaxation on relaxation oscillation of injection lasers," Trans. IEICE Japan, vol. E-62, pp. 241-245, 1979.
[18] Y. Suematsu, Ed., Semiconductor Lasers and Integrated Optics (in Japanese). Tokyo, Japan: Ohmsha, 1984

[19] G. A. Aclet, W. Nijman, and H.'t Lam, "Electron lifetime and diffusion constant in germanium-doped gallium arsenide," J. Appl. Phys., vol. 45, pp. 3033-3035, 1974.

[20] Y. Suematsu and K. Furuya, "Theoretical spontaneous emission factor of injection lasers," Trans. IEICE Jpn., vol. 60, pp. 464-472, 1977.

[21] T. Baba, T. Hamano, F. Koyama, and K. Iga, "Spontaneous emission factor of a microcavity DBR surface emitting laser," IEEE J. Quantum Electron., vol. 27, pp. 1347-1358, June 1991.

[22] M. Yamada and Y. Suematsu, "Analysis of gain suppression in undoped injection lasers," J. Appl. Phys., vol. 52, pp. 2653-2664, 1981.

[23] M. Asada, "Intraband relaxation time in quantum-well lasers," IEEE J. Quantum Electron., vol. 25, pp. 2019-2026, Sept. 1989.

[24] L. A. Coldren and S. W. Corzine, Diode Lasers and Photonic Integrated Circuits. New York: Wiley, 1995.

[25] K. Nozaki, A. Nakagawa, D. Sano, and T. Baba, "Ultralow threshold and single-mode lasing in microgear lasers and its fusion with quasi-periodic crystals," IEEE J. Select. Topics Quantum Electron., vol. 9, 2003.

[26] B. E. Little and S. T. Chu, "Estimating surface-roughness loss and output coupling in microdisk resonators," Opt. Lett., vol. 21, pp. 1390-1392, 1996.

[27] B.-J. Li and P.-L. Liu, "Numerical analysis of microdisk lasers with rough boundaries," IEEE J. Quantum Electron., vol. 33, pp. 791-795, May 1997.

[28] R. Ushigome, M. Fujita, and T. Baba, "GaInAsP microdisk injection laser with benzocyclobutene polymer cladding and its athermal effect," Jpn. J. Appl. Phys., vol. 41, pp. 6364-6369, 2002.

[29] M. Fujita and T. Baba, "Proposal and FDTD simulation of whispering gallery mode microgear cavity," IEEE J. Quantum Electron., vol. 37, pp. 1253-1258, 2001.

[30] - , "Microgear laser," Appl. Phys. Lett., vol. 80, pp. 2051-2053, 2002.

[31] H.-Y. Ryu, S.-H. Kim, H.-G. Park, J.-K. Hwang, and Y.-H. Lee, "Squarelattice photonic band-gap single-cell laser operating in the lowest order whispering gallery mode," Appl. Phys. Lett., vol. 80, pp. 3883-3885, 2002.

[32] M. Loncar, T. Yoshie, A. Scherer, P. Gogna, and Y. Qiu, "Low-threshold photonic crystal laser," Appl. Phys. Lett., vol. 81, pp. 2680-2682, 2002.

[33] M. Fujita, R. Ushigome, and T. Baba, "Strain relaxation effect in microdisk lasers with compressively-strained quantum wells," Appl. Phys. Lett., vol. 80, pp. 1511-1513, 2002.

Toshihiko Baba (M'93) was born in Nagano Prefecture, Japan, on November 12, 1962. He received the B.E., M.E., and Ph.D. degrees from the Division of Electrical and Computer Engineering, Yokohama National University (YNU), Yokohama, Japan, in 1985, 1987, and 1990, respectively.

During his Ph.D. work, he had been engaged in antiresonant reflecting optical waveguides (ARROWs) and lightwave circuits. In 1990, he joined the Tokyo Institute of Technology as a Research Associate. From 1991 to 1993, he worked on the spontaneous emission behavior in vertical cavity surface emitting lasers (VCSEL) and achieved the room temperature continuous wave (RT-CW) operation of a long-wavelength VCSEL. In 1994, he became an Associate Professor of YNU and started the research on photonic crystals (PCs), for which he reported the first fabrication and characterization of an InP-based light emitters and a line defect waveguide at lightwave frequencies, and microdisk lasers (MDLs), for which he achieved the first RT-CW operation with the smallest cavity and the lowest threshold, discussed some modifications of the cavity structure into microgear or PC-like structure, and demonstrated a MDL-based near-field sensor. $\mathrm{He}$ also studied an enhancement of light-extraction efficiency and passivation techniques. His recent interests are in various cavities, lasers, and functional devices. He is also active on a deep-grating distributed Bragg reflector for short cavity lasers and $\mathrm{Si}$ photonics based on nanowire waveguides.

Prof. Baba is a Member of the Institute of Electronics, Information and Communication Engineers (IEICE), the Japan Society of Applied Physics, and the American Physics Society. He received the Niwa Memorial Prize in 1991, the Best Paper Award of Micro-Optic Conference in 1993 and 1999, the Paper Award and Academic Encouragement Award from IEICE in 1994, and the Marubun Research Encouragement Award in 2000.

Daisuke Sano was born in Shizuoka, Japan, on August 24, 1979. He received the B.E. degree from the Division of Electrical and Computer Engineering, Yokohama National University, Hodogayaku, Yokohama, Japan, in 2002, where he is currently pursuing the M.E. degree.

Mr. Sano is a Member of the Japan Society of Applied Physics (JSAP). 